\title{
Magnetic Tunnel Window's Imprint and Beyond
}

\author{
Juan J. Alonso ${ }^{a}$, Julio F. Fernández ${ }^{\mathrm{b}}$ \\ a Departamento Física Aplicada, Universidad de Málaga, Málaga, 29071 (Spain) \\ bICMA, CSIC and Universidad de Zaragoza, 50009-Zaragoza (Spain)
}

\begin{abstract}
We report results from Monte Carlo simulations of systems of magnetic dipoles that relax through quantum tunneling, much as $\mathrm{Fe}_{8}$ crystals at very low temperature. For short times, a hole develops in suitably defined magnetic field pseudo-distributions, which matches the shape of the tunnel window (TW). Much later, ordinary field distributions $P(h)$ develop similar holes if thermal energies are not much larger than the TW's energy. Still later, below the long-range ordering (LRO) temperature, $P(h)$ exhibits the signature of LRO.
\end{abstract}

Key words: quantum tunneling, magnetization processes, dipolar interactions, cooling history, dipole field distributions PACS: $75.45 .+\mathrm{j}, 75.50 . \mathrm{X} . \mathrm{x}$

Magnetic relaxation in crystals of singlemolecule magnets, such as $\mathrm{Fe}_{8}$, has recently become a subject of great interest. Experimentally observed relaxation at temperatures $T$ that are below some $10^{-2}$ of single-ion crystal anisotropy barrier energies $U$ is temperature independent, and is duly attributted to magnetic quantum tunneling (MQT) [1]. As explained in Ref. [2], hyperfine interactions with nuclear spins open up tunneling windows (TW's) of energy $\varepsilon_{w}$ that are comparable to magnetic dipolar energies. Then,

$\Gamma^{\prime}\left(\varepsilon_{h}\right) \simeq \Gamma \eta\left(\varepsilon_{h} / \varepsilon_{w}\right)$,

is the tunneling rate for spins at very low temperature, where $\Gamma$ is some rate, $\eta(x) \sim 1$ for $x \mid \lesssim 1, \eta(x) \sim 0$ for $x \gtrsim 1$, and $2 \varepsilon_{h}$ is the Zeeman energy change upon tunneling. From here on, times are given in terms of $\Gamma^{-1}$. The results reported here in Figs. 1, 2(a), and 3 are for $\eta(x)=1$ for $|x|<1$ and $\eta(x)=0$ for $|x|>1$; in Fig. 2 (b) we show results for $\eta(x)=\exp (-|x|)$. We shall refer to the former TW as flat and to the latter one as exponential. An unusual magnetiza-

Email addresses: jjalonso@uma.es (Juan J. Alonso), jff@pipe.unizar.es (Julio F. Fernández).

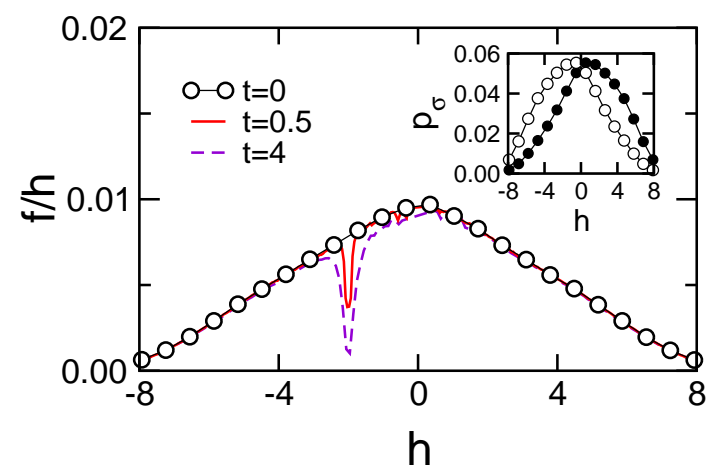

Fig. 1. $f / h$ versus $h$ for the shown times after quenching and applying a field $H=2$. All data points are for averages over $4 \times 10^{5}$ independent runs for $\varepsilon_{w}=0.1$. In the inset, $\bullet$ and $\circ$ are for $P_{+}$and $P_{-}$.

tion $M$ relaxation of fully polarized $\mathrm{Fe}_{8}$ like systems, $M_{t=0}-M_{t} \propto \sqrt{t}$, was predicted in Ref. [2] and later observed [3]. In an important piece of work, Wernsdorfer et al. [4] found that $M_{t} \propto \sqrt{t}$ if a small magnetic field $H$ is applied at $t=0$, after quenching from a higher temperature. This behavior has been explained recently [5]. In addition, since $d M_{t}(H) / d t=-2 \Gamma \int d h f(h, t) \eta(h+H)$ at all times, where $f(h, t)=\left[P_{+}(h, t)-P_{-}(h, t)\right]$, and $P_{+}(h)\left[P_{-}(h)\right]$ is the density distribution for fields 
$h$ acting on up [down] spins, $f(h, t)$ can be determined by a suitable application of magnetic fields. [ $f$ itself is not normalized, and is therefore not a distribution. In fact, it turns out that $f(h)$ is proportional to the system's energy [5]]. A "hole" of "intrinsic width", was thus observed to develop in time, and conjectured to correspond to the width in $\eta(h)$, i.e., to the width of the TW [4].

We report Monte Carlo simulations of Ising systems of interacting dipoles on SC lattices. Initially, spins are allowed to flip readily at temperature $T_{a} \gtrsim U / 10 \gtrsim T_{o}\left(T_{o} \simeq 2.5\right.$ is the LRO temperature) until the energy per spin reaches some value $\varepsilon_{a}$. A field $H$ is applied then and spins only flip at rate $\Gamma^{\prime}$ [see Eq. (1)] thereafter, thus simulating temperatures below some $10^{-2} U$. We have previously found [5] that $M_{t} \propto\left|\varepsilon_{a}\right| t$. Here, we report results for the evolution of the field pseudodistribution function $f(h)$ and for $p(H) \equiv P_{+}(h)+$ $P_{-}(h)$. Fields and energies are given in terms of nearest neighbor dipolar values.

How $P_{+}(h)$ and $P_{-}(h)$ split, leading to a nonvanishing $f(h)$, after thermalization at high $T$ is illustrated in the inset of Fig. 1. The hole that develops in $f(h)$ after quenching and applying field $H$ is shown in Fig. 1. The shape of the hole is shown in Figs. 2(a) and (b), for flat and exponential TW's, respectively, for $H=2$. Clearly, not only is the hole's width the same as the width of $\eta$, as conjectured in Ref. [4], but $f(h) / h$ matches $\eta(h)$ for the three shortest times shown in Figs. 2(a) and (b). When $t \gtrsim 1$, the hole widens, as shown in Fig. 2(a) for $t=4$.

On the time scale on which the hole in $f / h$ develops, $P(H)$ remains approximately independent of time. On the other hand, a hole which resembles the TW grows at much later times in $P(H)$ if $T \lesssim$ $\varepsilon_{w}[6]$. This is exhibited in Fig. 3. Still later, below the long-range ordering (LRO) temperature, $P(h)$ exhibits the signature of LRO [7] whether $T \lesssim \varepsilon_{w}$ or not. If, on the other hand, the energy of the system is kept constant, no LRO ensues, as expected. This is illustrated in Fig. 3.

\section{References}

[1] C. Sangregorio et al., Phys. Rev. Lett. 78, 4645 (1997).

[2] N. V. Prokof'ev and P. C. E. Stamp, Phys. Rev. Lett. 80, 5794 (1998).

[3] T. Ohm, C. Sangregorio, and C. Paulsen, Eur. Phys. J. B 6, 195 (1998).

[4] W. Wernsdorfer et al., Phys. Rev. Lett. 82, 3903 (1999).

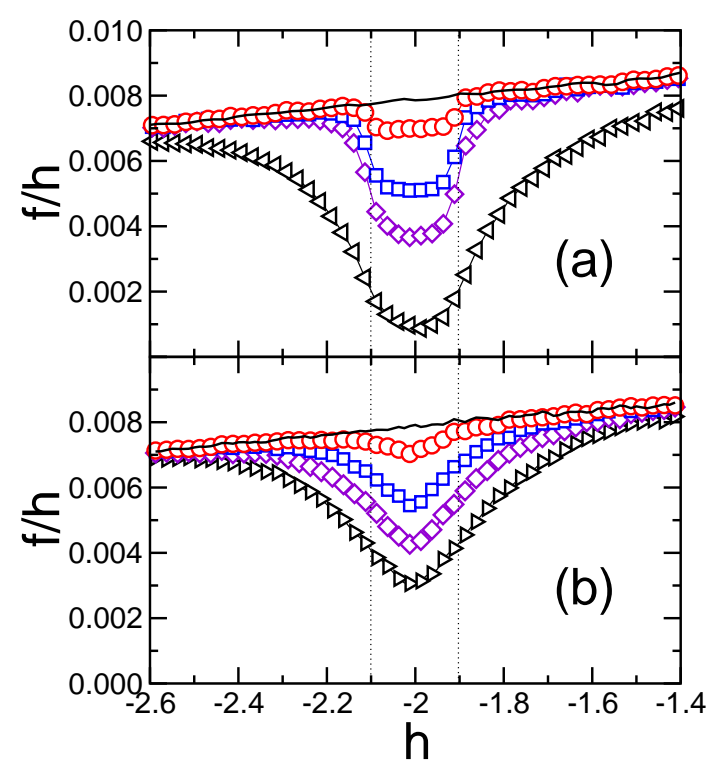

Fig. 2. (a) $f / h$ versus $h$ for short times with a flat TW of $\varepsilon_{w}=0.1, H=2$ and $\varepsilon_{a}=-0.51$ (b) Same as in (a) but for an exponential, TW with $\varepsilon_{w}=0.1$. The solid line, $\circ, \square, \diamond$ and $\triangleleft$ are for $t=0,0.0625,0.25,0.5$ and 4 , respectively.

[5] J. F. Fernández and J. J. Alonso, Phys. Rev. Lett. (in press).

[6] J. J. Alonso and J. F. Fernández, Phys. Rev. Lett. 87, 097205 (2001).

[7] J. F. Fernández, Phys. Rev. B 66, 064423 (2002).

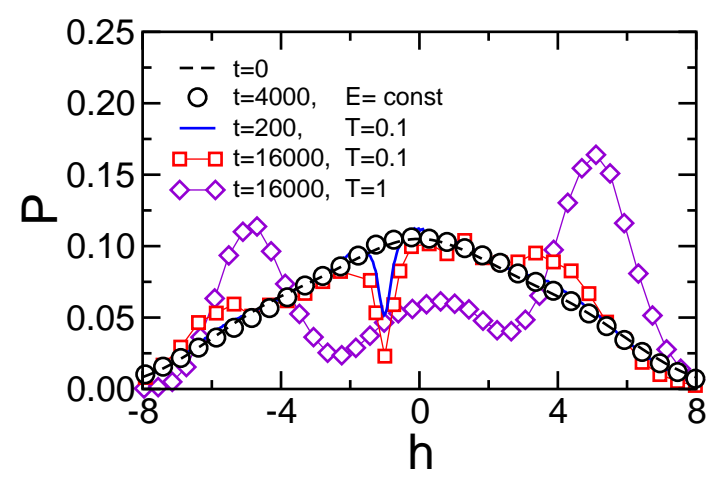

Fig. 3. The field distribution $P$ versus $h$ for the shown times, and $T=0.1$ or constant energy. In all cases $\varepsilon_{a}=-0.58, \epsilon_{w}=0.2$ and $H=1$. 